\title{
BMJ Open The specification, acceptability and effectiveness of respite care and short breaks for young adults with complex healthcare needs: protocol for a mixed- methods systematic review
}

Gerlinde Pilkington, ${ }^{\oplus 1}$ Katherine Knighting, ${ }^{\oplus 2}$ Lucy Bray, ${ }^{\oplus 2}$ Julia Downing, ${ }^{\circledR 3,4}$ Barbara A Jack, ${ }^{\odot 2}$ Michelle Maden, ${ }^{\odot 5}$ Ceu Mateus, ${ }^{\oplus}$ Jane Noyes, ${ }^{\oplus 7}$ Mary R O'Brien, ${ }^{\circledR 2}$ Brenda Roe, ${ }^{\circledR 2}$ Anthony Tsang, ${ }^{\circ}$ Sally Spencer ${ }^{\oplus}$

To cite: Pilkington $\mathrm{G}$, Knighting K, Bray L, et al. The specification, acceptability and effectiveness of respite care and short breaks for young adults with complex healthcare needs: protocol for a mixed-methods systematic review. BMJ Open 2019;9:e030470. doi:10.1136/ bmjopen-2019-030470

- Prepublication history and additional material for this paper are available online. To view these files, please visit the journal online (http://dx.doi. org/10.1136/bmjopen-2019030470).

Received 19 March 2019 Revised 24 April 2019 Accepted 13 May 2019
Check for updates

(C) Author(s) (or their employer(s)) 2019. Re-use permitted under CC BY-NC. No commercial re-use. See rights and permissions. Published by BMJ.

For numbered affiliations see end of article.

Correspondence to Gerlinde Pilkington; pilkingg@edgehill.ac.uk

\section{ABSTRACT}

Introduction The number of young adults with complex healthcare needs due to life-limiting conditions/complex physical disability has risen significantly as children with complex conditions survive into adulthood. Respite care and short breaks are an essential service, however, needs often go unmet after the transition to adult services, leading to a significant impact on the life expectancy and quality of life for this population. We aim to identify, appraise and synthesise relevant evidence to explore respite care and short breaks provision for this population, and to develop a conceptual framework for understanding service models.

Methods and analysis A mixed-methods systematic review conducted in two stages: (1) knowledge map and (2) evidence review. We will comprehensively search multiple electronic databases; use the Citations, Lead authors, Unpublished materials, Google Scholar, Theories, Early examples, and Related projects (CLUSTER) approach, search relevant websites and circulate a 'call for evidence'. Using the setting, perspective, intervention/phenomenon of interest, comparison and evaluation framework, two reviewers will independently select evidence for inclusion into a knowledge map and subsequent evidence review, extract data relating to study and population characteristics, methods and outcomes; and assess the quality of evidence. A third reviewer will arbitrate where necessary. Evidence will be synthesised using the following approaches: quantitative (narratively/conducting meta-analyses where appropriate); qualitative (framework approach); policy and guidelines (documentary analysis informed approach). An overall, integrated synthesis will be created using a modified framework approach. We will use Grading of Recommendations Assessment, Development and Evaluation (GRADE)/GRADE-Confidence in the Evidence from Reviews of Qualitative Research to assess the strength and confidence of the synthesised evidence. Throughout, we will develop a conceptual framework to articulate how service models work in relation to context and setting.

Ethics and dissemination Ethical approval is not required as this is a systematic review. We will present our
Strengths and limitations of this study

- The first systematic review of all available evidence on the nature and provision of respite and short break services for young adults with complex healthcare needs (CHCNs).

- Aims and review questions were developed with stakeholders from the advisory group including young adults with $\mathrm{CHCNs}$, parents and professionals.

- Use of a mixed-methods approach to enable inclusion of all types of qualitative, quantitative and policy evidence.

- Development of a knowledge map to characterise current services and a conceptual framework to inform future service provision and further research.

- There may be limited quantitative and health economic data from which to draw firm conclusions.

work in academic journals, at appropriate conferences; we will disseminate findings across networks using a range of media. Steering and advisory groups were established to ensure findings are shared widely and in accessible formats.

PROSPERO registration number CRD42018088780.

\section{INTRODUCTION}

Young adults with life-limiting conditions (LLCs) and young adults with complex physical disabilities often live with multiple comorbidities due to their complex healthcare needs (CHCNs). Care for these young adults is an ongoing complex process, with no simple care pathway, and often multiple, unplanned episodes of illness. The number of children with CHCNs who survive to become young adults is rising annually. ${ }^{2}$ In 2010, there were 55721 young adults with complex needs living in England $^{3}$ and an estimated 100000 disabled children with complex care 


\begin{tabular}{ll}
\hline Table 1 Key definitions \\
$\begin{array}{ll}\text { CHCNs } & \begin{array}{l}\text { Substantial and ongoing healthcare needs, typically across multiple health concerns, requiring a } \\
\text { coordinated response from more than one service }\end{array} \\
\begin{array}{l}\text { Complex physical } \\
\text { disability }\end{array} & \begin{array}{l}\text { Impairments and/or physical disabilities, due to congenital or acquired physical disability, or major } \\
\text { neurological trauma, that requires a complex level of physical management and support }\end{array} \\
\text { LLCs } & \begin{array}{l}\text { A life-limiting or life-threatening condition where there is no reasonable hope of cure and from which } \\
\text { the person is expected to die }\end{array} \\
\text { Respite care and short } \\
\text { breaks }\end{array}$ & $\begin{array}{l}\text { The temporary provision of formal (paid) or informal (unpaid) physical, emotional, spiritual or social } \\
\text { care for a dependent person, defined as follows. Formal respite care is provided by organisations } \\
\text { or individuals who receive financial payment, including family carers paid through management of } \\
\text { personal care budgets. Informal respite care does not involve financial payment }\end{array}$ \\
\hline
\end{tabular}

CHCNs, complex healthcare needs; LLCs, life-limiting conditions.

needs in England in 2007. ${ }^{4}$ This growing population require appropriate services to meet healthcare needs as they transition from children to adult services, including respite care and short breaks which are an essential component of support for young adults with CHCNs and their families. ${ }^{56}$ Table 1 details the key definitions used in this systematic review protocol.

Respite care and short breaks are beneficial to the person receiving care, their carers and families; for example increasing family carer resilience ${ }^{7}$ improving psychological well-being of parents, ${ }^{5} 8$ reducing risk of carer breakdown, ${ }^{79}$ and avoiding costly unplanned hospital admissions, length of stay or social care intervention. ${ }^{1011}$ Inadequate provision of services for young adults transitioning to adult care has a significant impact on life expectancy and quality of life, and increases the psychosocial burden on families and carers. ${ }^{12-15}$ Seven out of 10 families who care for someone with profound or multiple disabilities have reached, or come close to, 'breaking point' due to lack of short break services. ${ }^{16}$

In children services, short breaks provide opportunities for children to enjoy social interaction, support for family carers and support for siblings. ${ }^{17}$ Examples include residential schools, sitting services, day care in the home or other settings, or packages tailored to individual needs. ${ }^{18}$ In adult services, planned respite or replacement care focuses on support for carers rather than for the person receiving care. Typically, adult services meet the needs of older people with cancer or other terminal diagnoses, and may therefore be inappropriate for young adults with fluctuating health conditions, such as those with CHCNs. ${ }^{59131920}$ Limited respite care, particularly for those with very CHCNs, is available for planned short breaks or emergency family situations once young adults with CHCNs have transitioned to adult services. ${ }^{721} 22$

Despite the rising number of young people with CHCNs surviving into early adulthood and the consequent increase in service demand, the current scale, cost and types of available respite care have not been collated and systematically evaluated. The optimum service model for the provision of respite care and short breaks is currently uncertain; therefore, a systematic review of the available evidence is needed to inform the development of future services and to identify research priorities.

\section{OBJECTIVES}

The aims of this systematic review are to identify, appraise and synthesise evidence on the specification, acceptability, effectiveness and facilitators or barriers to respite care and short breaks provision for young adults with CHCNs due to a LLC or complex physical disability. The specific objectives are as follows:

1 . To identify and characterise the different types of formal and informal respite care and short break provision for young adults (18-40 years) with CHCNs due to a LLC or complex physical disability.

2. To determine the effectiveness and cost-effectiveness of different types of formal and informal respite care and short break provision for young adults (18-40 years) with CHCNs due to a LLC or complex physical disability.

3. To better understand the impact, experiences and perceptions of respite care and short break provision from the perspectives of service users and providers.

4. To explore current UK policy, not-for-profit-organisation (NFPO) publications and guideline recommendations regarding respite care and short break provision for young adults (18-40 years) with CHCNs due to a LLC or complex physical disability.

5. To develop a conceptual framework that shows the programme logic and articulates the programme theories of respite care and short break models for young adults (18-40 years) with CHCNs due to a LLC or complex physical disability that will inform service planning and commissioning.

6. To make recommendations for further empirical research to inform intervention development and evaluation.

\section{METHODS}

\section{Patient and public involvement}

A patient and public involvement advisory group (PAG) of young adults and parents has supported development 
of the systematic review protocol, including the systematic review questions and key definitions to facilitate the process being relevant, accessible, accountable and acceptable. ${ }^{23} 24$ The group communicates through a variety of methods to fit with the needs of individuals including face-to-face meetings, email, telephone and video communication. The PAG will continue to collaborate with the review team at key points throughout the study including contextualisation of the findings and dissemination plan. ${ }^{25}$ The steering group includes the review team, external professionals, representatives from national stakeholder organisations and two PAG representatives, including a young adult who is the co-chair.

\section{Design}

The overall design is a results-based, convergent synthesis, utilising a mixed-methods systematic review design: quantitative and qualitative data will be synthesised and presented separately, with a further synthesis of the two data types undertaken to create a third, integrated synthesis. ${ }^{26}$ The review methods are described in accordance with guidelines (Preferred Reporting Items for Systematic Reviews and Meta-Analyses (PRISMA) Protocols)) for the reporting of protocols of systematic reviews. ${ }^{27}$ We have adopted a two-stage approach for this mixed-methods systematic review to encompass the broad review questions and facilitate stakeholder involvement, based on methods in similar evidence syntheses. ${ }^{28}{ }^{29}$ The review processes are shown in figure 1.

The following review questions focus on young adults (18-40 years) with CHCNs:

1. What types of respite care and short breaks are provided in the UK and similar global economies?

2. What is the effectiveness and cost-effectiveness of different types of formal and informal respite care and short break provision?

3. What is the economic impact of respite care and short breaks?

4. What are service users' and providers' views of current service provision and the need for new services?

5. What are the facilitators and barriers to providing, implementing, using and sustaining respite care and short breaks, taking into account the different perspectives of service users, family members and providers?

6. What are the current UK policy and guidance recommendations for the provision of respite care and short breaks?

In Stage 1, we will identify, categorise and describe the evidence to create a knowledge map of different service typologies of respite care and short breaks for young adults with CHCNs and to identify gaps in the evidence base. The overall principle guiding development of the Stage 1 knowledge map is to be inclusive to ensure identification of all relevant evidence.

Evidence identified in Stage 1 will be considered for inclusion in Stage 2, where we will use appropriate methods to synthesise data and consider the methodological quality of the included evidence. We will construct an evidence matrix by service typology and type of evidence (effectiveness, cost-effectiveness, experience and attitudes, and policy and guidelines). We will extract key study characteristics, assess evidence quality and narratively synthesise information using appropriate tools and techniques. We will use the knowledge map and evidence synthesis to develop a conceptual framework of respite care provision for young adults with CHCNs.

\section{Eligibility criteria}

The setting, perspective, intervention/phenomenon of interest, comparison and evaluation (SPICE) framework $^{30}$ underpins the overall approach to searching for and selecting relevant evidence for inclusion, detailed in table 2. We have selected broad criteria to reflect the diversity of service provision and will include evidence from any study design that meets the SPICE criteria. We will identify qualitative, quantitative, and policy-related output as defined by and reported in each study, for any follow-up duration.

\section{Information identification \\ Search sources}

We will search the following electronic databases from 2002 to current: ASSIA (ProQuest), British Nursing Index (NICE Evidence Services, HDAS), CINAHL (EBSCO), Cochrane Central Register of Controlled Trials (Cochrane Library), Cochrane Database of Systematic Reviews (Cochrane Library), Database of Abstracts of Reviews of Effects (Cochrane Library), EMBASE (NICE Evidence Services, HDAS), Google Scholar, HMIC (NICE Evidence Services, HDAS), Joanna Briggs Institute COnNECT+, MEDLINE (OVID), NHS Economic Evaluations Database (Cochrane Library), NIHR Journals Library, PROSPERO, PsycINFO (EBSCO), Social Care Online, TRIP database, Web Of Science (Clarivate Analytics). We will search the following trials registries: International Clinical Trials Registry Platform, EU Clinical Trials Register and Clinical Trials.gov.

We will also search for grey and unpublished literature in Open Grey and Grey Literature Report, and search charity and organisational websites (eg, Together for Short Lives, PaedPalLit and WHO). We will use the Citations, Lead authors, Unpublished materials, Google Scholar, Theories, Early examples, and Related projects (CLUSTER) approach to identify additional outputs (eg, 'sibling' papers or 'kinship' studies) from the included evidence. ${ }^{31}$ Finally, we will circulate a 'call for evidence' via social media channels and networks/experts identified by the team, steering group and PAG.

We will limit the searches to evidence published from 1st January 2002 due to changes in patient population, service provision and policy change over the last 15 years. ${ }^{3}$ We will include only UK-specific evidence written in English language for the policy and guideline evidence; where feasible we will include non-English evidence for other streams. All available evidence will be included in 
Review Questions

1) What types of respite care and short breaks are provided in the UK and similar global economies?

2) What is the effectiveness and cost-effectiveness of different types of formal and informal respite care and short break provision?

3) What is the economic impact of respite care and short breaks?

4) What are service users and providers views of current service provision and the need for new services?

5) What are the facilitators and barriers to providing, implementing, using and sustaining respite care and short breaks, taking into account the different perspectives of service users, family members and providers?

6) What are the current UK policy and guidance recommendations for the provision of respite care and short breaks?

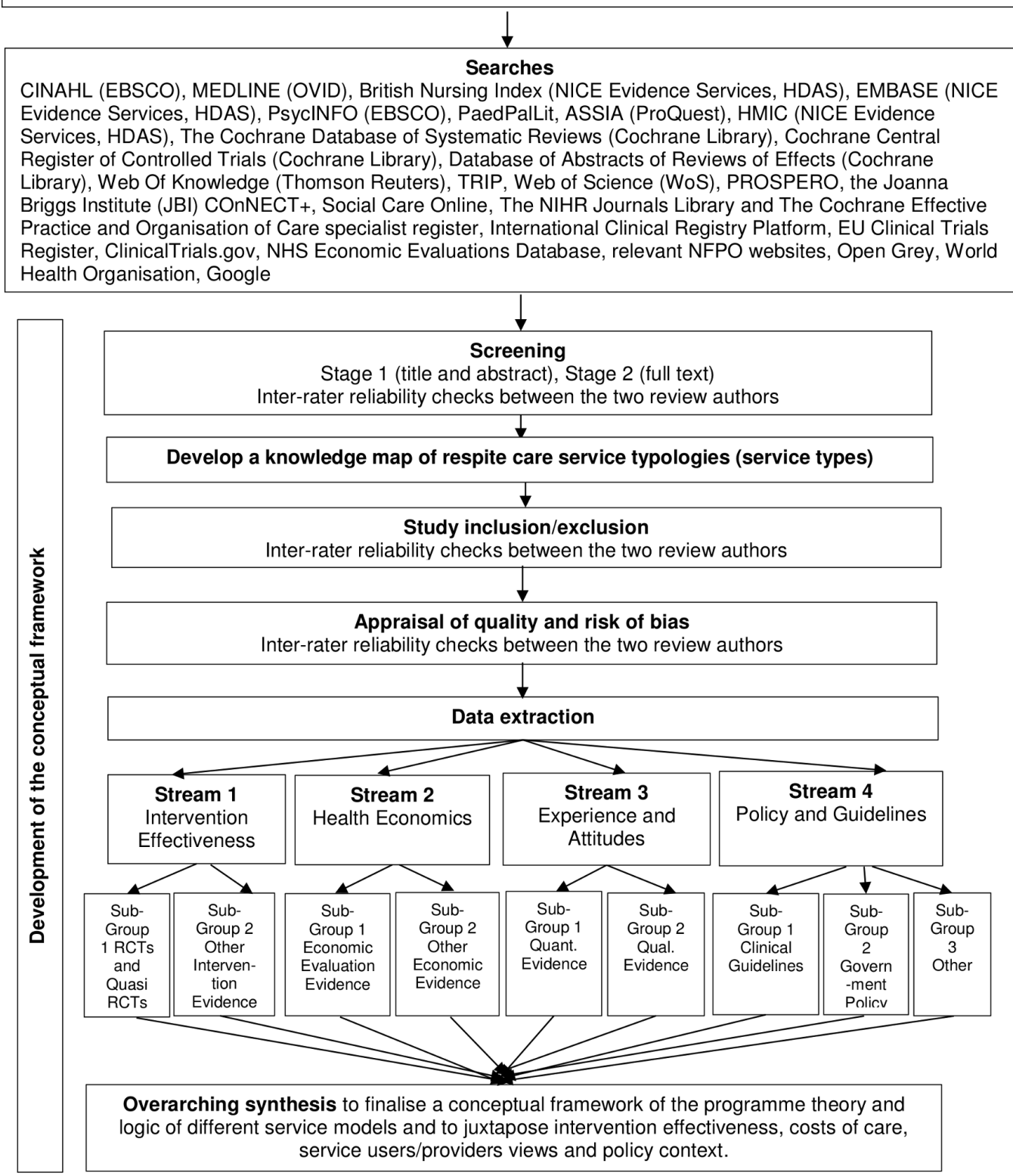

Figure 1 Review processes.

Stage 1 knowledge map but only evidence relevant to UK service provision will be included in Stage 2 .

\section{Search strategy}

An experienced information specialist will develop tailored search strategies with the review team, steering group and PAG; a MEDLINE search strategy will be developed using keywords, free-text terms and controlled vocabulary (online supplementary appendix 1). The
MEDLINE search strategy will then be translated into other databases.

\section{Study records}

Evidence selection

Search results will be de-duplicated and uploaded to Covidence, web-based systematic review management software. ${ }^{32}$ Two reviewers will independently screen all titles and abstracts using the inclusion criteria outlined 
Table 2 Inclusion criteria

\begin{tabular}{|c|c|c|}
\hline & Inclusion & Exclusion \\
\hline Setting & $\begin{array}{l}\text { Services and providers of formal respite care and/ } \\
\text { or short breaks (hospices, residential care homes, } \\
\text { adult day services, individual providers and paid } \\
\text { carers/family carers working in home settings, } \\
\text { informal care from unpaid family members, holiday } \\
\text { care) }\end{array}$ & $\begin{array}{l}\text { Services and providers of care other than } \\
\text { respite care and short breaks } \\
\text { Services specifically commissioned for young } \\
\text { adults with learning disability or mental health } \\
\text { needs }\end{array}$ \\
\hline $\begin{array}{l}\text { Intervention/ } \\
\text { phenomenon of interest }\end{array}$ & $\begin{array}{l}\text { Formal (paid) and informal (unpaid) respite care/ } \\
\text { short breaks }\end{array}$ & Care other than respite care and short breaks \\
\hline
\end{tabular}

CHCNs, complex healthcare needs; OECD, Organisation for Economic Cooperation and Development; QALY, quality-adjusted life year.

in table 2. For Stage 1 knowledge map, we will only use the perspective and intervention components of the SPICE criteria for evidence selection, that is, respite care or short breaks for young adults (18-40 years) with LLCs and/or complex physical disability.

We will only include evidence from mixed populations where (a) data from young adults is reported separately to those $<18$ or $>40$ years and (b) data from those with CHCNs, LLCs or complex physical disability are reported separately to those with other conditions.

Disagreements will be resolved through discussion and consultation within the review team. We will contact study authors for further information where eligibility is unclear. We will tabulate reasons for study exclusion and bibliographic details of evidence excluded at the full-text stage will be available on request as an electronic addendum. Results of the searching, mapping and selection processes will be reported for both stages using the PRISMA guidelines, including a flow diagram of included/excluded evidence. ${ }^{33}$

\section{Data extraction}

Bespoke piloted data extraction forms will be used to extract information from included evidence. In Stage 1, we will extract bibliographic, population and intervention details, using the Template for Intervention Description and Replication checklist as a guide. ${ }^{34}$

In Stage 2, two reviewers will independently extract the following information for each type of evidence (effectiveness, cost-effectiveness, evidence on experience and attitudes, and policy and guidelines):

- Publication characteristics: for example, year, dates and country of data collection, language, source of funding;

- Methods: for example, study design, duration of follow-up; 


\begin{tabular}{lll}
\hline Table 3 Methodological quality assessment tools & \\
\hline Experimental & Randomised controlled trial & Cochrane RoB tool \\
& Non-randomised controlled trial & Cochrane RoB tool EPOC adaptations for different study designs \\
& Before and after study & Cochrane RoB tool or National Institutes of Health tool \\
Observational & Cohort & CASP for cohort studies \\
& Case-control & CASP for case control studies \\
& Cross-sectional & CEBMa tool \\
& Interrupted time-series & Cochrane RoB (EPOC adaptation) \\
& Economic evidence & CEBMa tool \\
Qualitative & Qualitative & British Medical Journal Checklist for authors and peer reviewers of \\
Mixed-methods & Mixed-methods & CASP for qualitative studies \\
\hline Policy & Policy/guideline document & Appraisal of Guidelines, Research and Evaluation \\
\hline Other & Grey literature & Appropriate method-specific tool for the type of evidence. If a position \\
& & statement use the Authority, Accuracy, Coverage, Objectivity, Date, \\
\end{tabular}

CASP, Critical Appraisal Skills Programme; CEBMa, Centre for Evidence-Based Management; EPOC, Effective Practice and Organisation of Care; RoB, Risk of Bias.

- Aims, objectives, hypotheses, target audience;

- Participant characteristics: for example, type and duration of CHCNs, inclusion/exclusion criteria, age range, gender distribution, ethnicity, number in each study group, baseline characteristics, loss to follow-up;

- Types of care: for example, care provider (formal or informal), carer status (healthcare professional or not), care setting, duration of care

- Key limitations of each item of evidence;

- Description of all outcomes and their reported results.

Disagreements will be resolved through consensus and arbitration through a third reviewer where required. We will contact study authors to resolve uncertainties in study reports.

\section{Assessment of methodological quality}

Two reviewers will independently assess the quality and methodological limitations of included evidence using appropriate tools (see table 3), including experimental, observational, qualitative, and mixed-methods study designs, and policy/guidelines evidence. ${ }^{35-45}$ Disagreements will be arbitrated by a third reviewer until consensus is reached.

\section{Data synthesis methods}

In Stage 1, we will classify the evidence and consider factors such as population, timing and location to create a knowledge map of the different service typologies of respite care. Service typologies will be determined broadly by type, eligibility criteria and target population based on the data extracted to determine how they are intended to work, what they aim to achieve, what outcomes they include and for whom (programme theory) and to describe their programme logic (ie, components and processes in place to achieve the outcomes). Through consensus, existing knowledge and scoping searches, we have identified five preliminary categories of respite and short breaks: planned residential care; day care; homebased care; emergency care and holiday care (figure 2). The classification system may be revised following completion of the knowledge map in Stage 1.

In Stage 2, we will categorise selected evidence by service typology and evidence type (see figure 3 ). We anticipate an uneven distribution of the evidence and may need to implement a sampling frame to ensure that there is a representative sample of conditions. We will summarise service characteristics and the quality of the evidence for each type of service. We anticipate considerable variability within each service typology and across each evidence stream due to the nature of respite care, research methods and reporting. In the first instance, we will therefore discuss the findings for each aspect of the evidence matrix and refine the planned syntheses accordingly. We will record and report deviations from this published protocol.

\section{Evidence of effectiveness}

Data from randomised, quasi-randomised controlled trials or other intervention studies (eg, before and after studies or observational studies) will be tabulated and synthesised narratively by service type. We anticipate that meta-analyses will not be possible due the heterogeneous nature of the evidence. However, where appropriate we will conduct meta-analyses to estimate the effects of the intervention for each outcome, in accordance with recommendations in the Cochrane Handbook for Systematic Reviews of Interventions. ${ }^{35}$ Where data are sufficient, 

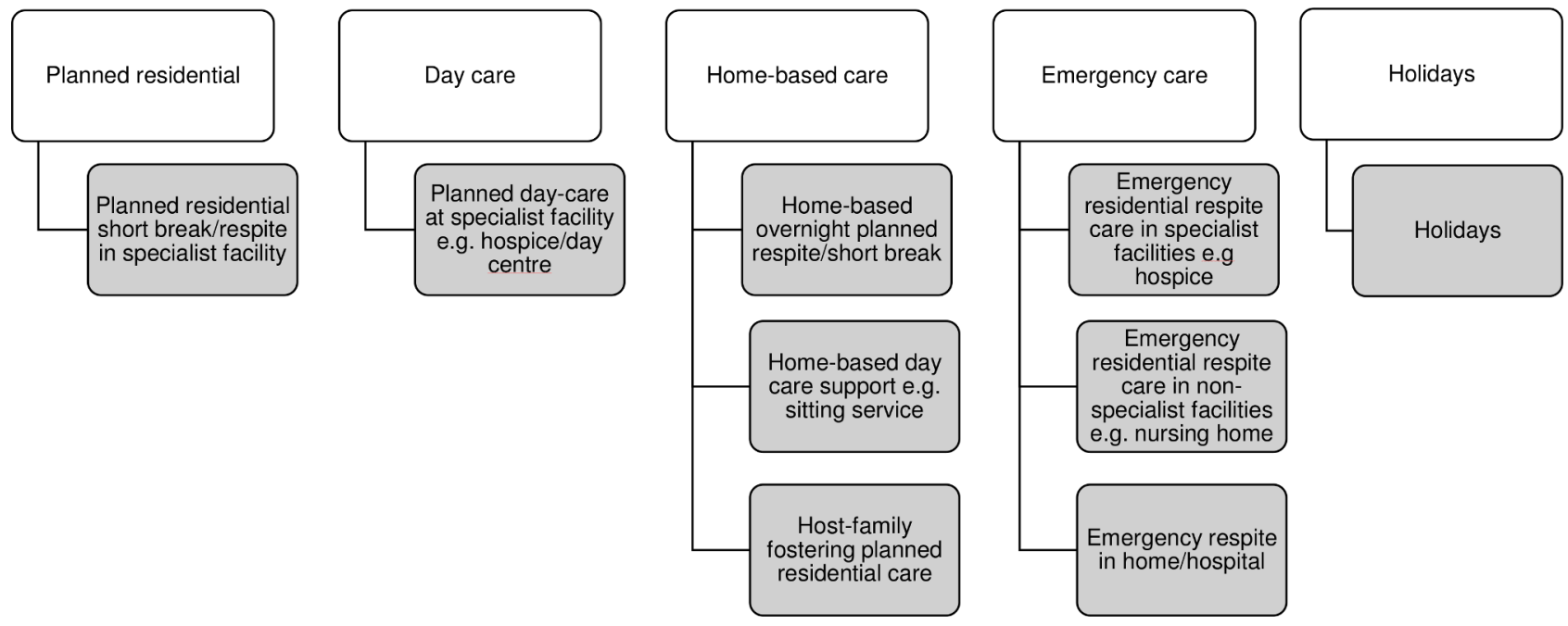

Figure 2 Initial types of respite care.

we will conduct sensitivity analyses based on missing data and risk of bias criteria (randomisation). Analyses will be conducted using Review Manager (RevMan) V.5. ${ }^{46}$
Evidence of cost-effectiveness

We will tabulate and narratively synthesise data derived from economic evaluations (eg, cost-utility and cost-effectiveness, reports of care costs) and other economic evidence (eg, cost of illness or burden of disease studies) by service type. We will consider all direct and indirect

Figure 3 Evidence matrix. 
costs of respite care (eg, direct medical and non-medical costs and productivity losses) including quality adjusted life-year, admission avoided and carer burden, taking account of the following factors: population size, service type, perspective (eg, patient/National Health Service and social services/societal), price year and currency, time horizon, discount rate and type of health-related quality of life instrument, where applicable.

\section{Evidence on experience and attitudes}

We will include and narratively report the results from qualitative, quantitative and mixed-methods evidence (eg, surveys, interviews, focus groups, observational studies, case studies, process evaluations). Quantitative data will be synthesised using the same methods as for effectiveness outcomes; qualitative data will be synthesised using framework synthesis. ${ }^{47}$ Findings from the qualitative and qualitative syntheses will be integrated using established methods for combining mixed-methods data. ${ }^{48}$

\section{Evidence from policy and guidelines}

The purpose of this evidence is to create framework within which we will contextualise the included evidence. We will conduct content analysis of the evidence from relevant current UK Government policy, clinical guidelines and NFPO literature using a documentary analysis informed approach ${ }^{49}$ to tabulate the evidence based on an a priori framework, following the process outlined for textual analysis. ${ }^{50}$

\section{Subgroup analyses}

Where possible, we will conduct subgroup analyses using the PROGRESS and PROGRESS-plus frameworks (place of residence, race/ethnicity, occupation, gender, religion, education, socioeconomic status, and social capital, age, disability and sexual orientation), endorsed by the Campbell and Cochrane Equity Methods Group for systematic reviews. ${ }^{51}$ We will explore subgroups of interest where data permits, for example, differences in outcomes between young adults who have transitioned from paediatric to adult services, and young adults who developed CHCNs in adulthood.

\section{Overall synthesis}

We will use the Evidence for Policy and Practice Information and Co-ordinating Centre framework method to integrate evidence across the evidence matrix. ${ }^{52}{ }^{53}$ Using an a priori framework, we will conduct within service type and evidence stream integration of qualitative and quantitative data based on the review questions. ${ }^{52}$ Experienced team members will lead the process to ensure that there are appropriate skills to synthesise mixed-methods evidence, and we have assigned arbitrators to mediate disagreements and uncertainties. We will consider the overall impact of methodological quality on the results by removing evidence at high risk of bias and comparing the output.

\section{Overall assessment of evidence}

Two reviewers will use the Grading of Recommendations Assessment, Development and Evaluation (GRADE) or Confidence in the Evidence from Reviews of Qualitative Research (GRADE-CERQual) ${ }^{54} 55$ systems to assess and report strength of the evidence. GRADE assesses the following domains: risk of bias, directness of the evidence, precision of effect estimates and risks of publication bias. GRADE-CERQual assesses methodological limitations, relevance to the review question, coherence of study findings and adequacy of the data. Results will be tabulated in summary of findings tables.

\section{Conceptual framework}

We will develop and refine a conceptual framework of respite care and short break provision for young adults with CHCNs throughout Stages 1 and 2. The programme theory (what they aim to achieve, what outcomes they include and for whom) and programme logic (components and processes in place to achieve the outcomes) for each service type developed in Stage 1, along with findings from Stage 2 will be explored through discussion within the review team, steering group and PAG to produce a conceptual framework. We will use Cochrane guidance $^{56}$ and examples of good practice ${ }^{57-59}$ to guide the final programme theories and logic models for the different types of respite care and produce the final conceptual framework. The framework will contain the most important components from Stages 1 and 2 findings to ensure that the concepts and relationships are clear and useful for researchers, commissioners, service providers, and policymakers to inform future research and service development.

\section{REVIEW REPORTING}

There are no guidelines for reporting mixed-method reviews, so we will follow Cochrane guidance to select relevant reporting elements from method-specific reporting guidelines such as PRISMA for quantitative evidence and enhancing transparency in reporting the synthesis of qualitative research for qualitative evidence. ${ }^{3360}$

\section{ETHICS AND DISSEMINATION}

As this is a systematic review of published literature, ethics approval is not required. A dissemination and pathway to impact plan has been developed in conjunction with stakeholders and the PAG. Outputs including the knowledge map, results of the review and conceptual framework will be shared with all relevant audiences through a range of networks and using a variety of media. In addition, we will present findings at key conferences and publish in peer-reviewed journals.

Author affiliations

${ }^{1}$ Postgraduate Medical Institute (PGMI), Faculty of Health \& Social Care, Edge Hill University, Ormskirk, UK 
${ }^{2}$ Research and Innovation Team, Faculty of Health \& Social Care, Edge Hill University, Ormskirk, UK

${ }^{3}$ International Children's Palliative Care Network, Assagay, South Africa

${ }^{4}$ Edge Hill University, Ormskirk, UK

${ }^{5}$ Liverpool Reviews and Implementation Group, University of Liverpool, Liverpool, UK ${ }^{6}$ Division of Health Research, Faculty of Health and Medicine, Lancaster University, Lancaster, UK

${ }^{7}$ School of Health Sciences, Bangor University, Bangor, UK

Contributors All authors (GP, KK, LB, JD, BAJ, MM, CM, JN, MRO'B, BR, AT and SS) have contributed to the development of all aspects of the protocol. The review design and methodology were developed by GP, LB, JD, BAJ, MM, CM, JN, MRO'B, $B R$, the chief investigator SS and project manager KK. MM and GP developed the search strategies, AT contributed to the scoping of preliminary types of respite and short breaks. This manuscript was drafted by GP, with contributions from KK, LB, JD, BAJ, MM, CM, JN, MRO'B, BR, AT, SS and all authors have critically revised the manuscript and approved the final version and act as guarantors of the review.

Funding The review is funded by the National Institute for Health Services Research (NIHR), Health Services \& Delivery Research (HS\&DR) programme (Study ID 16/115/17). The study is sponsored by Edge Hill University.

Disclaimer The views and opinions expressed therein are those of the authors and do not necessarily reflect those of the HS\&DR Programme, NIHR, NHS or the Department of Health.

Competing interests None declared.

Patient consent for publication Not required.

Provenance and peer review Not commissioned; peer reviewed for ethical and funding approval prior to submission.

Open access This is an open access article distributed in accordance with the Creative Commons Attribution Non Commercial (CC BY-NC 4.0) license, which permits others to distribute, remix, adapt, build upon this work non-commercially, and license their derivative works on different terms, provided the original work is properly cited, appropriate credit is given, any changes made indicated, and the use is non-commercial. See: http://creativecommons.org/licenses/by-nc/4.0/.

\section{REFERENCES}

1. Burns KH, Casey PH, Lyle RE, et al. Increasing prevalence of medically complex children in US hospitals. Pediatrics 2010;126:638-46.

2. Fraser LK, Miller M, Hain R, et al. Rising national prevalence of lifelimiting conditions in children in England. Pediatrics 2012;129:e92 3-e929.

3. Fraser LF, Miller M, Aldridge J, et al. Prevalence of life-limiting and life-threatening conditions in young adults in England 2000-2010 http://www.togetherforshortlives.org.uk/assets/0000/6736/TFSLAdul tReport2013Final.pdf

4. Equality act 2010. 2010 http://www.legislation.gov.uk/ukpga/2010/ 15/pdfs/ukpga 20100015 en.pdf (Accessed 28 Feb 2018).

5. Remedios C, Willenberg L, Zordan R, et al. A pre-test and post-test study of the physical and psychological effects of out-of-home respite care on caregivers of children with life-threatening conditions. Palliat Med 2015;29:223-30.

6. Together for Short Lives. A guide to the development of children's palliative care services. http://www.togetherforshortlives.org. uk/assets/0001/1649/ACT_Guide_to_Developing_Services.pdf (Accessed 28 Feb 2018).

7. Mitchell T, Knighting K, O'Brien M R JBR. Short break and emergency respite care: an exploration of options for children and young people with life-limiting conditions when hospice is unavailable. Int J Palliat Nurs 2016;22:57-65.

8. Whiting M. Support requirements of parents caring for a child with disability and complex health needs. Nurs Child Young People 2014;26:24-7.

9. Carter B, Edwards M, Hunt A. 'Being a presence'. Journal of Child Health Care 2015;19:304-19.

10. Ling J. Respite support for children with a life-limiting condition and their parents: a literature review. Int J Palliat Nurs 2012;18:129-34.

11. Robertson J, Hatton $C$, Wells $E$, et al. The impacts of short break provision on families with a disabled child: an international literature review. Health Soc Care Community 2011;19:337-71.

12. Choices NHS. Carers' breaks and respite care - NHS.UK. 2015 https://www.nhs.uk/conditions/social-care-and-support/breaks-forcarers-respite-care/ (Accessed 28 Feb 2018).
13. Doug M, Adi Y, Williams J, et al. Transition to adult services for children and young people with palliative care needs: a systematic review. Arch Dis Child 2011;96:78-84.

14. National Collaboration for Integrated Care and Support. National Collaboration for Integrated Care and Support. https://www.gov.uk/ government/uploads/system/uploads/attachment_data/file/198748/ DEFINITIVE_FINAL_VERSION_Integrated_Care_and_Support_-_Our Shared_Commitment_2013-05-13.pdf (Accessed 17 Mar 2018).

15. Marsh S, Cameron M, Duggan M, et al. Young people with lifelimiting conditions: transition to adulthood. 'Small numbers, huge needs, cruel and arbitrary division of services'. Executive Summary of Phase 1 report for Marie Curie Cancer Care. 2011 https://www. mariecurie.org.uk/globalassets/media/documents/commissioningour-services/past-initiatives/ypt/phase1_young-people-transitionexec-summary.pdf (Accessed 17 Mar 2018)

16. Foundation for people with learning disabilities. Learning disabilty statistics: effects of being a carer. 2014 www.learningdisabilities.org. uk/help-information/LearningDisability-Statistics-/187702

17. Together for Short Lives. Children's palliative care definitions. http:// www.togetherforshortlives.org.uk/assets/0000/4089/CPC_definitions. pdf (Accessed 28 Feb 2018).

18. Mooney A, Owen C, Statham J, et al. Disabled Children: Numbers, Characteristics and Local Service Provision. http://discovery.ucl.ac. uk/1482347/1/Numbers_and_characteristics_of_disabled_children. pdf (Accessed 28 Feb 2018).

19. Kirk S, Fraser C. Hospice support and the transition to adult services and adulthood for young people with life-limiting conditions and their families: a qualitative study. Palliat Med 2014;28:342-52.

20. Fraser LK, Aldridge J, Manning S, et al. Hospice provision and usage amongst young people with neuromuscular disease in the United Kingdom. Eur J Paediatr Neurol 2011;15:326-30.

21. Noyes J, Pritchard A, Rees S, et al. Bridging the gap: transition from children's to adult palliative care. 2014 https://www.bangor. ac.uk/so/bridging-the-gap/Final Report Bridging the Gap between children $\% 27 \mathrm{~s}$ and adult palliative care 10-14.pdf.

22. Care Quality Commission. From the pond into the sea. Children's transition to adult health services. Gallowgate, 2014.

23. Harris J, Croot L, Thompson J, et al. How stakeholder participation can contribute to systematic reviews of complex interventions. $J$ Epidemiol Community Health 2016;70:207-14.

24. Jamal F, Langford R, Daniels $\mathrm{P}$, et al. Consulting with young people to inform systematic reviews: an example from a review on the effects of schools on health. Health Expectations 2015;18:3225-35.

25. Tripp D. Critical incidents in action inquiry. Shaklock G, Being reflexive in critical educational and social research. London: Falmer Press, 1998:36-49.

26. Harden A, Thomas J, Cargo M, et al. Cochrane Qualitative and Implementation Methods Group guidance series-paper 5: methods for integrating qualitative and implementation evidence within intervention effectiveness reviews. J Clin Epidemiol 2018;97:70-8.

27. Moher D, Shamseer L, Clarke M, et al. Preferred reporting items for systematic review and meta-analysis protocols (PRISMA-P) 2015 statement. Syst Rev 2015;4:1-19.

28. Thomas J, Sutcliffe K, Harden A, et al. Children and healthy eating: a systematic review of barriers and facilitators, 2003.

29. Whitaker R, Hendry M, Aslam R, et al. Intervention Now to Eliminate Repeat Unintended Pregnancy in Teenagers (INTERUPT): a systematic review of intervention effectiveness and cost-effectiveness, and qualitative and realist synthesis of implementation factors and user engagement. Health Technol Assess 2016;20:1-214.

30. Booth A. Clear and present questions: formulating questions for evidence based practice. Library Hi Tech 2006;24:355-68.

31. Booth A, Harris J, Croot E, et al. Towards a methodology for cluster searching to provide conceptual and contextual "richness" for systematic reviews of complex interventions: case study (CLUSTER). BMC Med Res Methodol 2013;13:118.

32. Veritas Health Innovation. Covidence systematic review software. http://www.covidence.org.

33. Moher D, Liberati A, Tetzlaff J, et al. Preferred reporting items for systematic reviews and meta-analyses: the PRISMA statement. PLoS Med 2009;6:e1000097.

34. Hoffmann TC, Glasziou PP, Boutron I, et al. Better reporting of interventions: template for intervention description and replication (TIDieR) checklist and guide. BMJ 2014;348:g1687.

35. Higgins JPT, Green S. Cochrane Handbook for Systematic Reviews of Interventions. Version 5.1.0. Updated 2011;2011 http://handbook. cochrane.org.

36. Cochrane Effective Practice and Organisation of Care (EPOC). Suggested risk of bias criteria for EPOC reviews, 2016:1-3. http:// epoc.cochrane.org/sites/epoc.cochrane.org/files/public/uploads/ 
Resources-forauthors2017/suggested_risk_of_bias_criteria_for_ epoc_reviews.pdf

37. Brouwers MC, Kho ME, Browman GP, et al. AGREE II: advancing guideline development, reporting and evaluation in health care. Can Med Assoc J 2010;182:E839-E842.

38. National Heart, Lung, and Blood Institute (NHLBI). Study quality assessment tools. https://www.nhlbi.nih.gov/health-topics/studyquality-assessment-tools (Accessed 13 Jul 2018).

39. Critical Appraisal Skills Programme (CASP). 12 questions to help you make sense of a Cohort Study How. https://casp-uk.net/wp-content/ uploads/2018/01/CASP-Cohort-Study-Checklist.pdf

40. Critical Appraisal Skills Programme (CASP). 11 questions to help you make sense of a Case Control Study. Case-Control Cohort Des. Framew. 2018 https://casp-uk.net/wpcontent/uploads/2018/01/ CASP-Case-Control-Study-Checklist.pdf

41. Center for Evidence-Based Management (CEBMa). Critical appraisal of a case study, 2016.

42. Center for Evidence-Based Management (CEBMa). Critical Appraisal of a Cross-Sectional Study (Survey). Web Page. 2016. 2014 https:// www.cebma.org/wp-content/uploads/CriticalAppraisal-Questionsfor-a-Cross-Sectional-Study-july-2014.pdf\%0Ahttps://www.cebma. org/

43. Drummond MF, Jefferson TO. Guidelines for authors and peer reviewers of economic submissions to the BMJ. BMJ 1996;313:275-83.

44. CASP. 10 questions to help you make sense of qualitative research, 2014.

45. Pluye P, Hong QN. Appraising qualitative, quantitative and mixed methods studies included in mixed studies reviews: The MMAT. Annu Rev Public Health 2014;35:29-45.

45. Review Manager (RevMan), 2014.

47. Oliver SR, Rees RW, Clarke-Jones L, et al. A multidimensional conceptual framework for analysing public involvement in health services research. Health Expect 2008;11:72-84.

48. Pluye P, Hong QN. Combining the power of stories and the power of numbers: mixed methods research and mixed studies reviews. Annu Rev Public Health 2014;35:29-45.
49. Bowen GA. Document analysis as a qualitative research method. Qualitative Research Journal 2009;9:27-40.

50. O'Leary Z. The essential guide to doing your research project. 2nd edn. Thousand Oaks, CA: SAGE Publications, Inc., 2014.

51. Welch V, Petticrew M, Tugwell P, et al. PRISMA-Equity 2012 extension: reporting guidelines for systematic reviews with a focus on health equity. PLoS Med 2012;9:e1001333.

52. Oliver S, Harden A, Rees R, et al. An emerging framework for including different types of evidence in systematic reviews for public policy. Evaluation 2005;11:428-46.

52. 53 EPPI-Centre. The Evidence for Policy and Practice Information, Social Science Research Unit. http://eppi.ioe.ac.uk/cms/ (Accessed 19 Mar 2018).

52. Cochrane Methods. GRADEing. GRADEPro GDT, 2015.

55. Lewin $\mathrm{S}$, Glenton $\mathrm{C}$, Munthe-Kaas $\mathrm{H}$, et al. Using qualitative evidence in decision making for health and social interventions: an approach to assess confidence in findings from qualitative evidence syntheses (GRADE-CERQual). PLoS Med 2015;12:e1001895.

56. Noyes J, Hendry M, Booth A, et al. Current use was established and Cochrane guidance on selection of social theories for systematic reviews of complex interventions was developed. J Clin Epidemiol 2016;75:78-92.

57. Bray N, Noyes J, Edwards RT, et al. Wheelchair interventions, services and provision for disabled children: a mixed-method systematic review and conceptual framework. BMC Health Serv Res 2014;14:1-18.

58. Anderson LM, Petticrew M, Rehfuess E, et al. Using logic models to capture complexity in systematic reviews. Res Synth Methods 2011;2:33-42.

59. Kneale D, Thomas J, Harris K. Developing and optimising the use of logic models in systematic reviews: exploring practice and good practice in the use of programme theory in reviews. PLoS One 2015;10:e0142187-26.

60. Flemming K, Booth A, Hannes K, et al. Cochrane Qualitative and Implementation Methods Group guidance series-paper 6: reporting guidelines for qualitative, implementation, and process evaluation evidence syntheses. J Clin Epidemiol 2018;97:79-85. 